
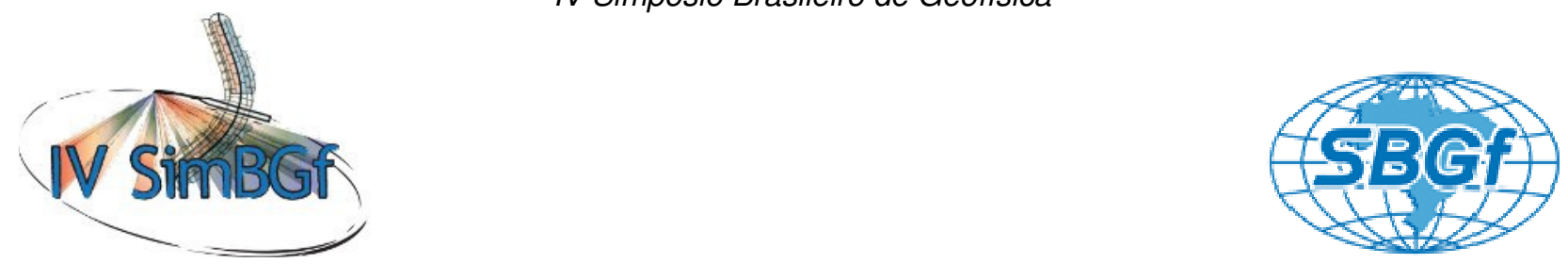

\title{
Estudo da Sismicidade na Região de Sobral - CE, NE do Brasil, em 2008
}

Paulo Henrique Sousa de Oliveira ${ }^{1}$, Joaquim Mendes Ferreira ${ }^{1}$, Aderson Farias do Nascimento ${ }^{1}$, Francisco Hilário Rego Bezerra $^{1}$, José Eduardo Soares ${ }^{2}$, Reinhardt Fuck ${ }^{2}$

1-PPGG/Universidade Federal do Rio Grande do Norte; 2-Instituto de Geociências/UnB

Copyright 2010, SBGf - Sociedade Brasileira de Geofísica

Este texto foi preparado para a apresentação no IV Simpósio Brasileiro de Geofísica, Brasília, 14 a 17 de novembro de 2010. Seu conteúdo foi revisado pelo Comitê Técnico do IV SimBGf, mas não necessariamente representa a opinião da SBGf ou de seus associados. É proibida a reprodução total ou parcial deste material para propósitos comerciais sem prévia autorização da SBG.

\section{Resumo}

No início de 2008, uma intensa atividade sísmica iniciouse nas proximidades de Sobral - CE, ocorrendo um evento com magnitude $4,2 \mathrm{~m}_{\mathrm{b}}$ no dia 21 de maio. Todos os eventos, desde o início, foram registrados pela estação SBBR, que opera na região desde agosto de 2007. Após o evento de maio de 2008, o estudo da atividade sísmica foi realizado com uma rede sismográfica local, constituída de até 11 estações sismográficas digitais. Neste estudo foram analisados os 581 sismos registrados, no mínimo, em três estações, objetivando a determinação de hipocentros e mecanismos focais. Para a determinação hipocentral, foi utilizado um modelo de semi-espaço homogêneo, com parâmetros iguais a $6,00 \mathrm{~km} / \mathrm{s}$ para a $\mathbf{V}_{\mathbf{P}}$ e 1,71 para $\mathbf{V}_{\mathbf{p}} / \mathbf{V}_{\mathbf{s}}$, obtidos a partir da análise dos dados. Foi encontrada uma zona sísmica ativa com profundidade variando entre $1 \mathrm{~km}$ e $8 \mathrm{~km}$, com $6 \mathrm{~km}$ de extensão na horizontal e com direção aproximada E-W. Com um conjunto de 24 sismos, com melhor precisão hipocentral obtivemos os parâmetros dos planos de falha e mecanismos focais, os quais foram do tipo transcorrente, com movimento dextral. Apesar da região possuir duas importantes estruturas tectônicas próximas ao local da área estudada, a falha Café-Ipueiras e o Lineamento Sobral-Pedro II, não foi possível obter correlação entre a sismicidade encontrada e qualquer uma dessas feições geológicas.

\section{Introdução}

A região Noroeste do Ceará é uma das principais regiões de atividade sísmica ativa do Nordeste do Brasil e possui um histórico de ocorrência de sismos relatado desde o século XIX no município de Granja, em 1810 (Ferreira \& Assumpção, 1983). Estudos de sismologia nessa região, realizados pelo Laboratório Sismológico da UFRN (LabSis), foram iniciados em 1988 após a ocorrência, no município de Groaíras, de dois sismos com magnitudes, respectivamente, de $4,0 \mathrm{~m}_{\mathrm{b}}$ e $3,9 \mathrm{~m}_{\mathrm{b}}$. Após estes eventos, várias campanhas têm sido realizadas para estudar a sismicidade ocorrida na região, com a instalação de redes locais, como por exemplo: 1) em Irauçuba e Hidrolândia, em 1991; 2) em Coreaú, Uruoca e Granja, em 1992; 3) em 1997, no município de Senador Sá e 4) na região de Sobral, em 2008, após a ocorrência de uma intensa atividade sísmica, iniciada no dia 28 de janeiro com um evento de magnitude $2,5 \mathrm{~m}_{\mathrm{b}}$, sendo sentido no distrito do Jordão, distrito de Sobral. A atividade continuou nos meses subseqüentes e o maior evento da sequência, de magnitude $4,2 \mathrm{~m}_{\mathrm{b}}$, ocorreu no dia 21 de maio. Essa sismicidade foi registrada, desde o seu início, pela estação sismográfica SBBR, a qual foi instalada em agosto de 2007 na região com recursos do Projeto do Instituto do Milênio "Estudos geofísicos e tectônicos na Província Borborema", e ainda está em operação na fazenda da EMBRAPA Caprinos.

Devido a ocorrência da sismicidade citada e de dois eventos ocorridos no dia 21 de maio de 2008 com uma diferença de apenas três minutos, alcançando magnitudes, respectivamente, $4,2 \quad m_{b}$ e $3,9 \quad m_{b}$, pesquisadores do LabSis decidiram instalar na região uma rede sismográfica local, que foi constituída de equipamentos do Laboratório de Estudos Litosféricos da UnB e estações de período curto do Projeto do Milênio (além da estação SBBR), composta por estações providas de sismômetros de período curto modelo S13J (vertical, NS e EW, em cada estação) e de estações broadband (banda larga) modelo KS2000M, todas com registrador SMART24(R).

\section{Resumo da Geologia da Área Estudada}

A região onde se encontra a atividade sísmica de Sobral, objeto deste estudo, está localizada no Domínio Médio Coreaú (DMC; Figura 1), que representa a margem Noroeste da Província Borborema (Almeida et al., 1981), pertencentes ao Nordeste do Brasil.

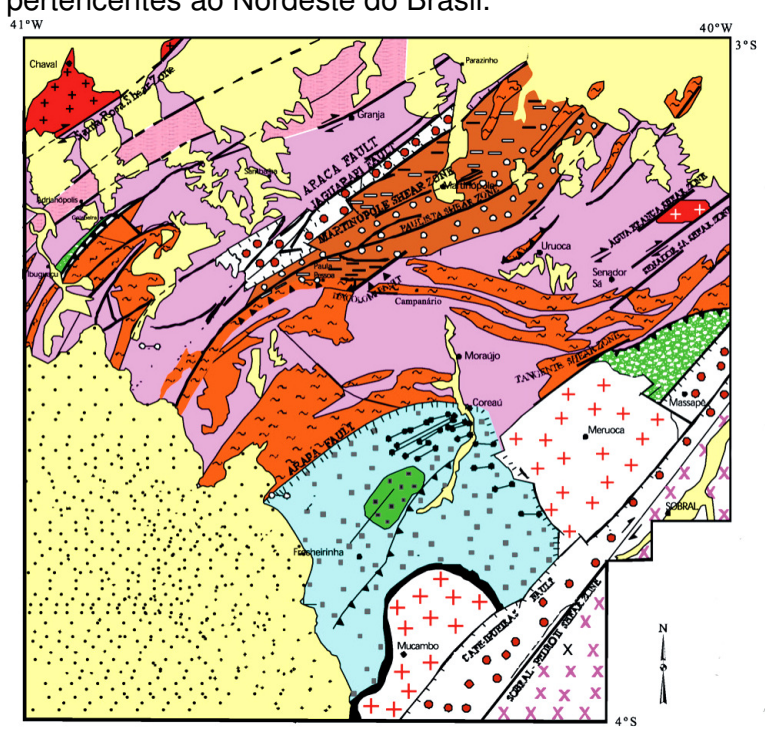

Figura 1 - Mapa geológico simplificado do Domínio Médio Coreaú (Modificado de Santos et al., 2008). 
O DMC é caracterizado, tectonicamente, por um sistema de zonas de cisalhamento Neoproterozóicas de direção NE - SW, destacando-se o Lineamento Sobral - Pedro II (LSPII). Este lineamento é um segmento de uma descontinuidade de magnitude continental: o Lineamento Transbrasiliano (LTB), o qual contorna o limite entre o Cráton Amazônico e a porção Leste da Plataforma Sul Americana (Feng et al., 2004).

\section{Metodologia}

A instalação da rede sismográfica de Sobral (SB) ocorreu em três etapas. A primeira etapa foi iniciada no começo de junho, sendo instaladas cinco estações, as quais operaram entre os dias 06 de junho de 2008 e 09 de julho de 2008. Na segunda etapa foram instaladas mais cinco, totalizando onze estações sismográficas digitais $\mathrm{e}$ abrangeu o período entre 10 de julho de 2008 e 26 de agosto de 2008. A terceira etapa constituiu na retirada de cinco estações. Assim, a rede SB voltou a atuar com seis estações sismográficas digitais. Esta configuração foi mantida entre 27 de agosto e 24 de setembro de 2008 . Todas estas etapas (Figura 2) possibilitaram aquisição de dados, objetivando a determinação de hipocentros e mecanismos focais compostos.

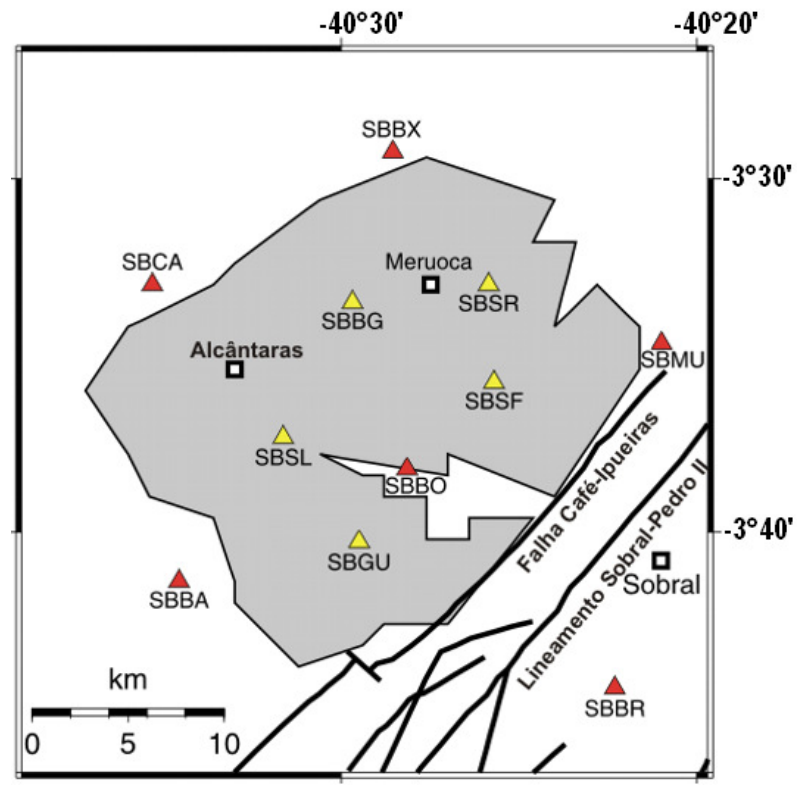

Figura 2 - Etapas de instalação da rede sismográfica de Sobral. Quadrados simbolizam as cidades, triângulos vermelhos as estações constituintes da primeira etapa. Todos os triângulos simbolizam as estações que constituíram a segunda etapa e os amarelos representam as estações constituintes da terceira etapa. As linhas grossas representam Zonas de cisalhamento, conforme DNPM (1973).

Durante todo o período de operação da rede SB, cerca de 2820 sismos foram registrados, em pelo menos uma estação. Neste artigo foram analisados os 581 sismos que foram registrados, no mínimo, em três estações. O programa SAC (Tapley \& Tull, 1991) foi utilizado para fazer a leitura dos tempos de chegada e das polaridades das ondas $\mathrm{P}$ e $\mathrm{S}$. Os hipocentros foram determinados com o programa HYPO71 (Lee \& Lahr, 1975), que tem sido utilizado com sucesso no estudo de sismicidade local no Nordeste do Brasil. A solução do mecanismo focal composto, obtida com auxílio do programa FPFIT (Reasemberg \& Oppenheimer, 1985) foi encontrada baseada no método que considera o primeiro movimento da onda $P$ e suas respectivas polaridades. O FPFIT considera que o padrão de radiação é do tipo duplobinário sem momento e busca numa malha de solução uma média ponderada de discrepâncias nas polaridades, considerando a variância estimada dos dados e a amplitude da radiação da onda $P$ (Reasemberg \& Oppenheimer, 1985). Como conseqüência do modelo de duplo-binário sem momento, temos um problema ambíguo, existindo dois planos nodais capazes de reproduzir o mesmo padrão de polaridades. Esta ambigüidade pode ser solucionada se existe conhecimento da distribuição hipocentral, que neste estudo servirá como suplemento para a construção dos mecanismos focais.

\section{Resultados}

A localização de sismos, para uma rede local, é baseada principalmente em um modelo de velocidade de camadas horizontais e o problema de variações laterais de velocidade normalmente é ignorado. Para encontrar o modelo de velocidades local, e os hipocentros, foi utilizado o programa HYPO71. Para determinar esse modelo, inicialmente é necessário encontrar um conjunto confiável de dados e, para isto, foi usado o diagrama de Wadati (Kissingler \& Engdahl, 1973).

\section{Diagrama de Wadati}

Entre os 581 sismos registrados em três ou mais estações, 157 foram registrados, no mínimo, em cinco estações, sendo utilizados para a execução do diagrama de Wadati. Durante a obtenção deste diagrama, foram descartadas as leituras acima do dobro do valor do desvio padrão, em relação a reta determinada por mínimos quadrados. Realizando isto sucessivamente, restou um conjunto confiável de 93 eventos e o coeficiente k obtido foi:

$$
\mathbf{k}=\frac{\mathbf{V}_{\mathbf{p}}}{\mathbf{V}_{\mathbf{S}}}=1,709 \pm 0,001
$$

\section{Modelo de Velocidades e Hipocentros}

O modelo de velocidades foi obtido de forma conjunta, considerando o Diagrama de Wadati como critério de consistência dos dados e escolhendo o conjunto de parâmetros com maior quantidade de sismos que tiveram as menores médias de erros. Isto foi feito variando $V_{P}$ de $5,0 \mathrm{~km} / \mathrm{s}$ a $6,4 \mathrm{~km} / \mathrm{s}$ (Charmichael, 1982; Bormann, 2002) e a razão $V_{\mathrm{P}} / V_{S}$ de 1,60 até 1,74 . Os valores médios do erro quadrático médio (RMS), a média do erro horizontal $(E R H)$ e a média do erro vertical (ERZ) foram calculadas com auxilio do programa HYPO71. O melhor modelo encontrado de forma conjunta foi: $V_{P}=6,00 \mathrm{~km} / \mathrm{s}$ e $V_{P} /$ $\mathbf{V}_{\mathbf{s}}=1,71$. A maioria dos trabalhos anteriores em sismologia, principalmente no Brasil, utilizaram o programa HYPO71, em particular no nordeste brasileiro (Ferreira et al., 1987, 1995, 1998; Takeya, 1992; do Nascimento, 1997; França, 1999; Vilar, 2000; Lima Neto, 
2009; Oliveira, 2010). Neste artigo também será utilizado o programa HYPO71 em um modelo de crosta em semi espaço infinito, homogêneo e isotrópico. O meio homogêneo nos fornece trajetórias de raios, provenientes das ondas $\mathrm{P}$ e $\mathrm{S}$, em forma de linhas retas. Este modelo torna-se aceitável, pois a região sísmica está localizada sobre embasamento Pré - Cambriano, o qual é constituído de rochas consolidadas e de baixa atenuação. Em geral, isto acarreta, nos sismogramas, chegadas das ondas $\mathrm{P}$ e S bem definidas (Figura 3 ).

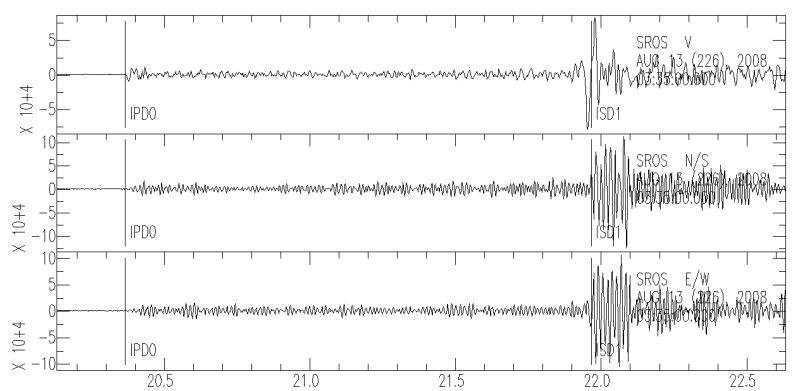

Figura 3 - Sismograma registrado na estação SBSR de um evento ocorrido em 13 de agosto de 2008, às 12h35min (hora local), registrado em sete estações da rede $S B$.

A ocorrência da atividade sísmica durante os quatro meses de operação da rede SB, com cerca de 2820 sismos, está mostrada na figura 3.

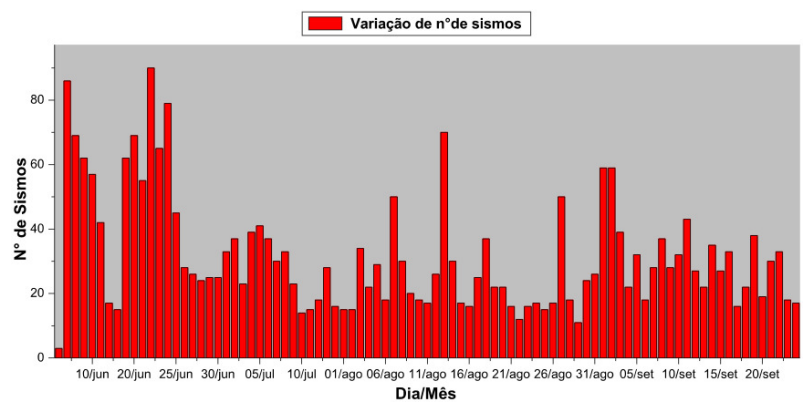

Figura 4 -Número de sismos por dia durante os quatro meses de operação da rede $S B$.

Como visto anteriormente, para a rede SB, a primeira etapa constituiu de seis estações sismográficas digitais, a segunda etapa de onze estações e a terceira etapa de seis estações sismográficas. Portanto serão mostrados os mapas epicentrais dos dados das três etapas separados.

\section{Primeira Etapa}

$\mathrm{Na}$ primeira etapa de atuação da rede SB foram registrados 1290 sismos. Entre estes, 237 sismos foram registrados, no mínimo, em três estações. O mapa epicentral obtido para esta etapa está representado pela figura 5 .

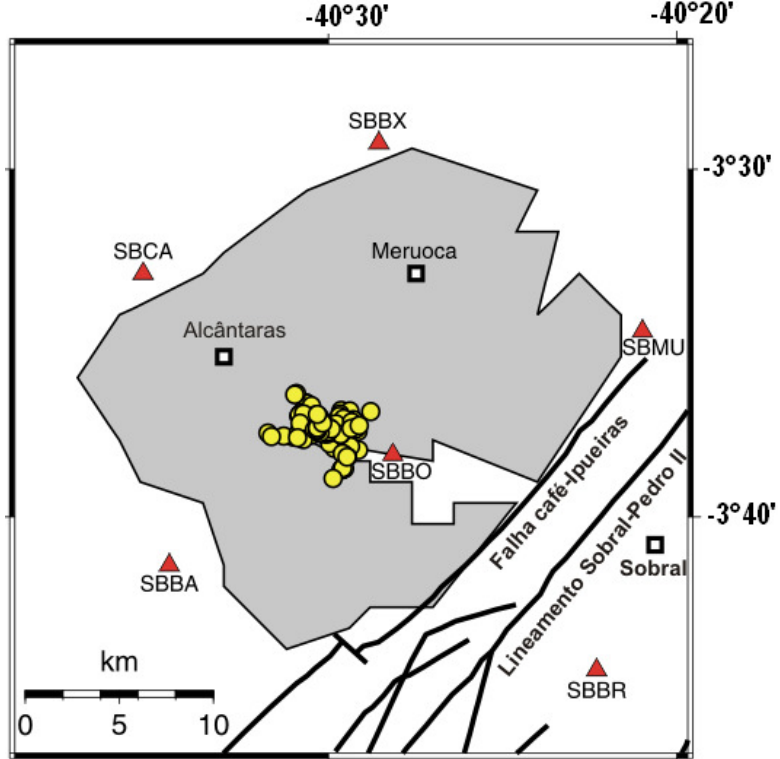

Figura 5 - Mapa epicentral dos 237 sismos registrados (representados por círculos amarelos), no mínimo, em três estações sismográficas, durante a primeira etapa de operação da rede $S B ;$ com os seguintes critérios: $R M S \leq 0,04 \mathrm{~s}$; $E R H \leq 0,3 \mathrm{~km} ; E R Z \leq 0,4 \mathrm{~km} ; N O$ (número de observações) $=10$. Demais símbolos de acordo com a figura 2.

\section{Segunda Etapa}

Nesta etapa de operação foram registrados 674 sismos. Entre estes, 312 sismos foram registrados, no mínimo, em três estações. O mapa epicentral obtido está representado pela figura 6 .

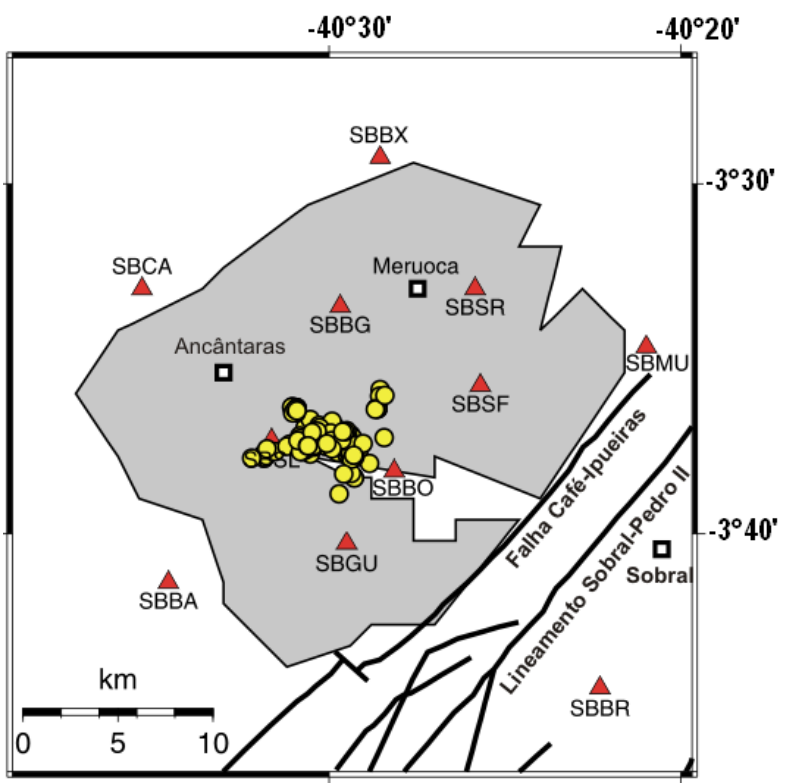

Figura 6 - Mapa epicentral dos 312 sismos registrados (representados por círculos amarelos), no mínimo, em três estações sismográficas, durante a segunda etapa de operação da rede $S B$, utilizando os seguintes critérios: $R M S \leq 0,04 s$; 
$E R H \leq 0,3 \mathrm{~km} ; E R Z \leq 0,4 \mathrm{~km} ; N O$ (número de observações) $=10$. Demais símbolos de acordo com a figura 2.

\section{Terceira Etapa}

$\mathrm{Na}$ terceira etapa de atuação da rede SB a atividade sísmica registrada na estação SBSL foi bastante alta, devido a sua proximidade dos epicentros. Neste período, 853 sismos foram registrados, onde apenas 32 sismos foram catalogados, no mínimo, em três estações. O mapa epicentral obtido está representado pela figura 7.

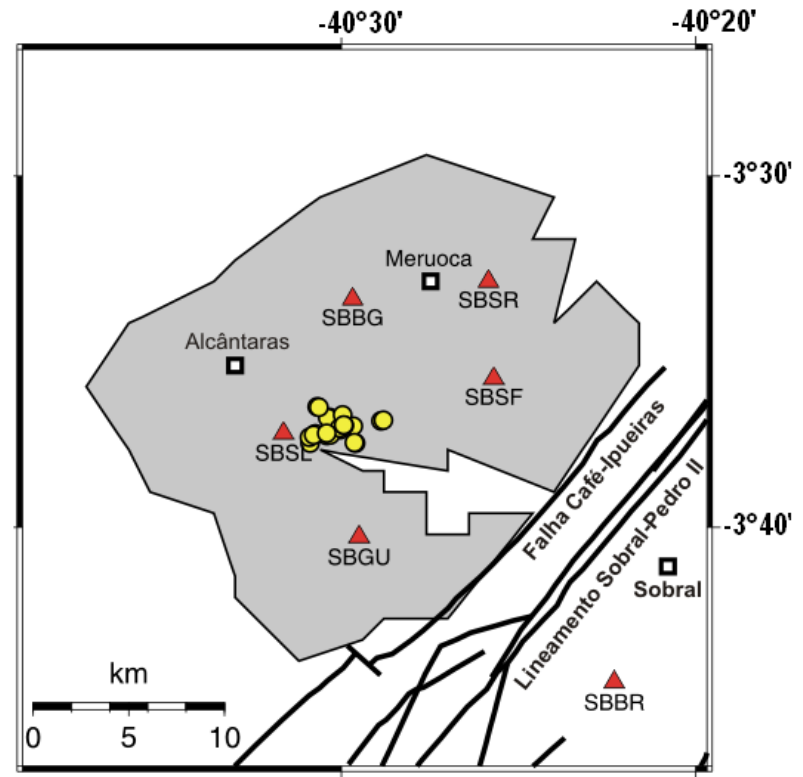

Figura 7 - Mapa epicentral dos 32 sismos registrados (representados por círculos amarelos), no mínimo, em três estações sismográficas, durante a terceira etapa de operação da rede $S B$, utilizando os seguintes critérios: $R M S \leq 0,04 s$; $E R H \leq 0,3 \mathrm{~km} ; E R Z \leq 0,4 \mathrm{~km} ; N O$ (número de observações) $=10$.

\section{Planos de Falha e Mecanismos Focais}

Com o objetivo de obter uma melhor visualização da distribuição epicentral e uma maior confiabilidade para a determinação dos parâmetros de falhamento, realizamos algumas filtragens nos dados obtidos para o modelo de velocidades, utilizando os seguintes critérios: $N O=10$, RMS $\leq 0,03 \mathrm{~s}, \mathrm{ERH} \leq 0,1 \mathrm{~km} ; \mathrm{ERZ} \leq 0,2 \mathrm{~km}$ e GAP $<180^{\circ}$. Realizando isso, foram selecionados 24 sismos para determinar os parâmetros de falhamento, mostrados na figura 8.

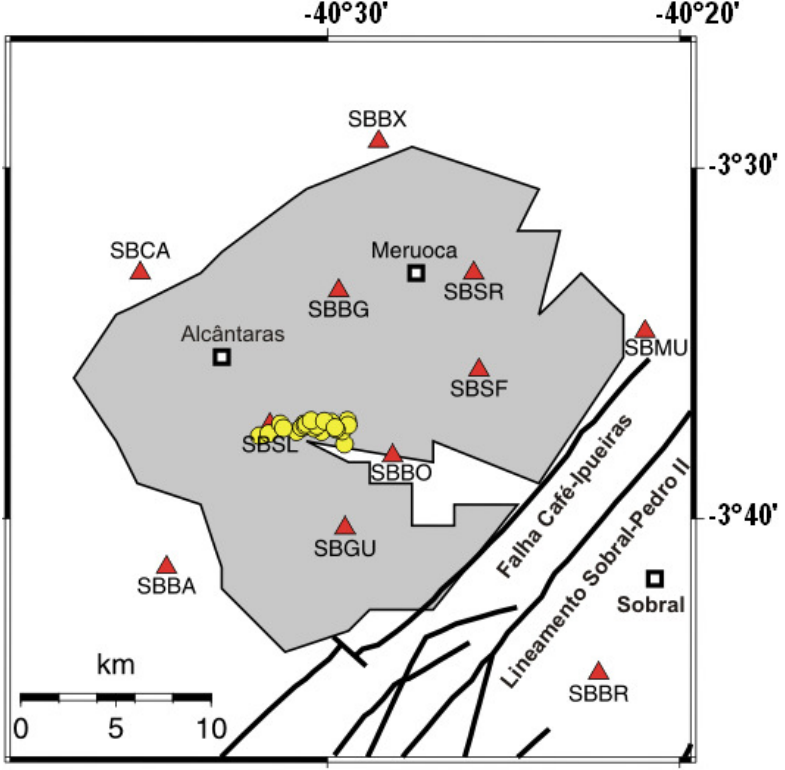

Figura 8 - Mapa epicentral dos 24 sismos selecionados, simbolizados por círculos amarelos, seguindo os critérios: $N O$ $=10, R M S=0,03 \mathrm{~s}, E R H \leq 0,1 \mathrm{~km} ; E R Z \leq 0,2 \mathrm{~km}$ e $G A P<180^{\circ}$.

Utilizando o método de mínimos quadrados para o ajuste do possível plano de falha, obtemos o azimute de $81^{\circ}$ (cf. correlação $=0,860$ ) para a direção desses 24 sismos. Com isso, construímos a figura 9, mostrando a projeção paralela e perpendicular ao possível plano de falha.
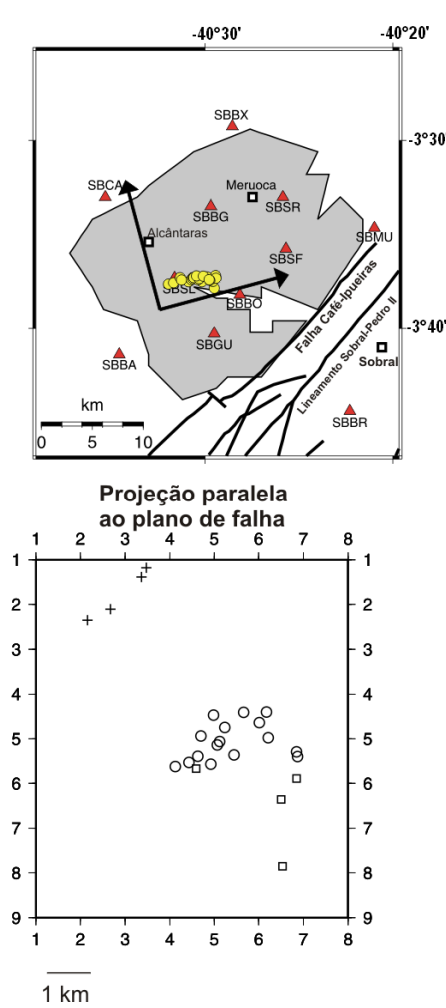

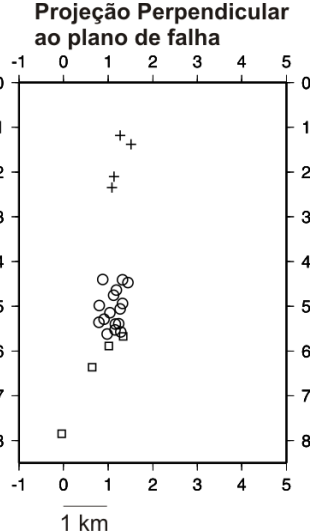

Legenda

$+\quad 1.1 \mathrm{~km}<\mathbf{h}<3.4 \mathrm{~km}$

$3.4 \mathrm{~km}<\mathbf{h} \leq 5.7 \mathrm{~km}$

$\square \quad 5.7 \mathrm{~km}<\mathbf{h} \leq 7.8 \mathrm{~km}$ 
Figura 9 - Mapa detalhado dos hipocentros da figura 8, com suas projeções em plano) perpendicular e paralelo ao possível plano de falha. $h$ representa profundidade. Esta projeção foi feita com um ponto central coincidindo com a origem das setas pretas. Demais símbolos de acordo com a figura 2.

O mecanismo focal composto para os 24 sismos selecionados, com direção de $81^{\circ}$, foi calculado e consistiu de grandes discrepâncias de polaridades, dificultando a visualização e interpretação. Considerando isso e a figura 9, foi proposta uma divisão em três grupos principais, constituídos de sismos com profundidade $(\mathrm{h})$ menor (1,1 km $<\mathrm{h}<3,4 \mathrm{~km}$, grupo A), com profundidade intermediária $(3,4 \mathrm{~km}<\mathrm{h} \leq 5,7 \mathrm{~km}$, grupo $\mathrm{B})$ e com sismos mais profundos $(5,7 \mathrm{~km}<\mathrm{h} \leq 7,8 \mathrm{~km}$, grupo $\mathrm{C})$. Então serão mostrados os mecanismos focais encontrados para cada grupo de sismos, fixando a direção encontrada com mínimos quadrados: $81^{\circ}$.

\section{Grupo A - 1,1 km $<\mathrm{h}<3,4 \mathrm{~km}$}

Usando o programa FPFIT foram obtidos os parâmetros mostrados na tabela 1. Para visualizar os resultados foi construída a figura 10.

Tabela 1 - Resultados obtidos com o programa FPFIT para o grupo A.

\begin{tabular}{lccc}
\hline & & & \\
& STRIKE & DIP & RAKE \\
\hline FPFIT & $81^{\circ}$ & $86^{\circ} \pm 5^{\circ}$ & $-170^{\circ} \pm 10^{\circ}$ \\
\hline
\end{tabular}

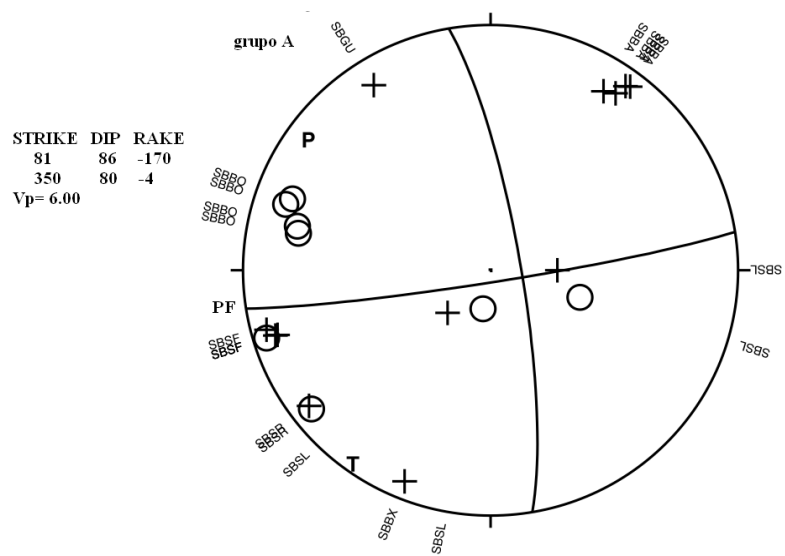

Figura 10 - Mecanismo focal composto para o grupo A obtido como auxílio do programa FPFIT. Hemisfério inferior, projeção de área igual. Cruzes e círculos representam, respectivamente, os eixos de compressão $(P)$ e de extensão $(T)$ e PF o plano de falha.

\section{Grupo B - 3,4 km $<\mathrm{h} \leq 5,7 \mathrm{~km}$}

Usando o programa FPFIT, foram obtidos os valores mostrados na tabela 2, sendo possível construir a figura 10.
Tabela 2 - Resultados obtidos com o programa FPFIT para o grupo $B$.

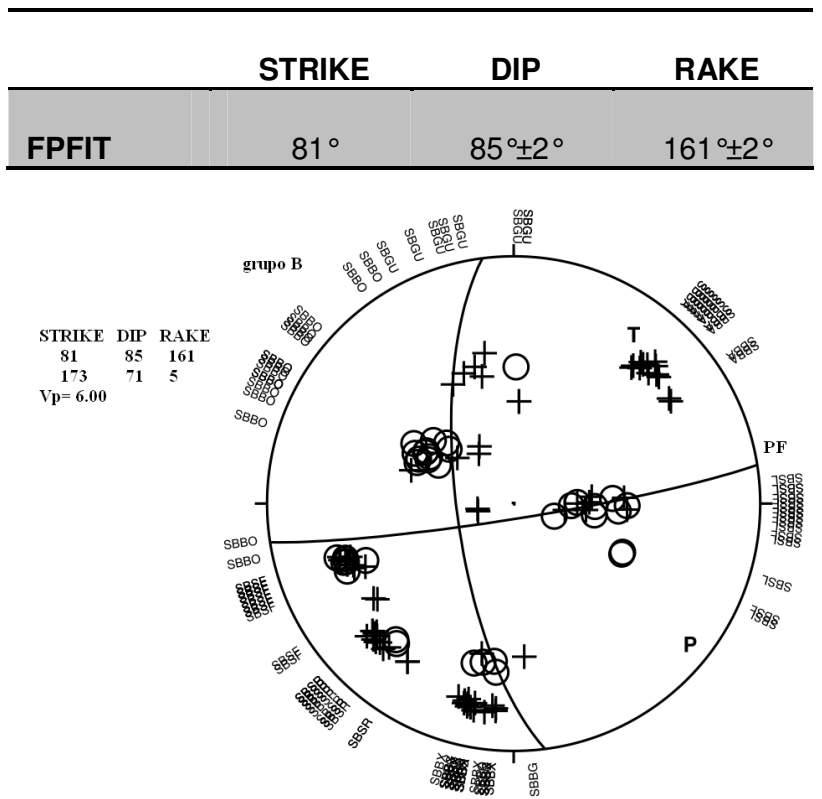

Figura 11 - Mecanismo focal composto para o grupo B obtido como auxílio do programa FPFIT. Hemisfério inferior, projeção de área igual. Cruzes e círculos representam, respectivamente, os eixos de compressão $(P)$ e de extensão $(T)$ e PF o plano de falha.

\section{Grupo $\mathrm{C}-\mathbf{5 , 7} \mathrm{km}<\mathrm{h} \leq 7,8 \mathrm{~km}$}

Usando o programa FPFIT, foram obtidos os parâmetros mostrados na tabela 3 , sendo possível construir a figura 12.

Tabela 3 - Resultados obtidos com o programa FPFIT para o grupo $C$

\begin{tabular}{lccc}
\hline & STRIKE & DIP & RAKE \\
\hline FPFIT & $81^{\circ}$ & $84^{\circ} \pm 1^{\circ}$ & $-164^{\circ} \pm 5^{\circ}$ \\
\hline
\end{tabular}




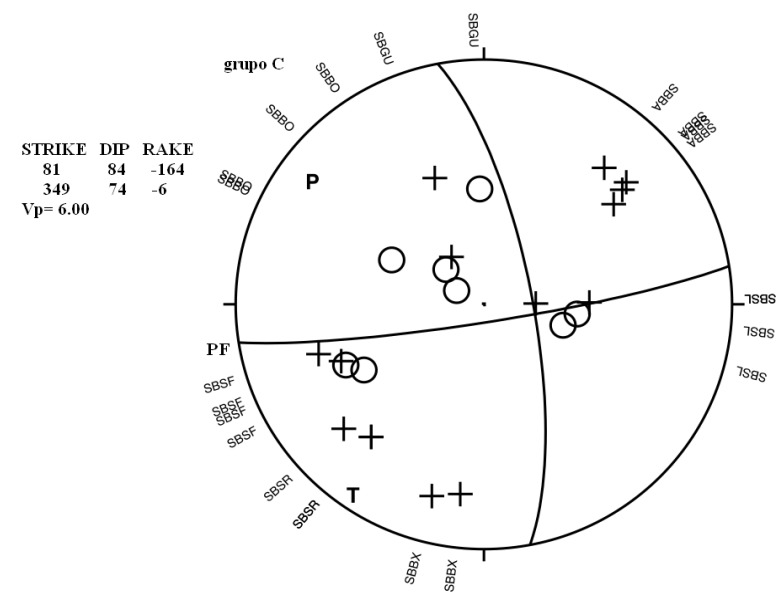

Figura 12 - Mecanismo focal composto para o grupo C obtido como auxílio do programa FPFIT. Hemisfério inferior, projeção de área igual. Cruzes e círculos representam, respectivamente, os eixos de compressão $(P)$ e de extensão $(T)$ e PF o plano de falha.

Como podemos observar nas figuras 10,11 e 12 todos os mecanismos focais possuem falhamento do tipo transcorrente, com movimento dextral.

\section{Discussão e Conclusões}

Com os dados registrados em cada uma das três etapas de operação da rede SB, obtivemos dados hipocentrais que revelaram várias distribuições, com diferentes direções (Figuras 5, 6 e 7). Estas premissas sugerem que outras falhas sismogênicas possam estar afetando o Granito Meruoca, onde a sismicidade estudada está concentrada. Com o objetivo de obter parâmetros hipocentrais mais precisos e gerar uma melhor visualização dos hipocentros e dos mecanismos focais, selecionamos um conjunto de 24 sismos com critérios especificados, sendo possível obter resultados significativos, como uma zona sísmica ativa com aproximadamente $6 \mathrm{~km}$ de extensão na horizontal, com direção aproximada E-W (Figura 8) e profundidade variando entre 1 e $8 \mathrm{~km}$. A falha sismogênica encontrada não possui diretamente correlação com as principais falhas mapeadas na região: a falha Café-Ipueiras e o Lineamento Sobral-Pedro II. O método de mínimos quadrados possibilitou obtermos a direção do possível plano de falha $\left(81^{\circ}\right)$ e comprovamos o fato que informações sobre distribuição hipocentral tornam mais simples a interpretação dos mecanismos focais, os quais revelaram falhamentos transcorrentes, sendo que este é o tipo de falhamento que predomina no Nordeste do Brasil (Ferreira et al., 1998). Os resultados encontrados mostram mais uma vez, que nem sempre é possível obter correlação entre sismicidade e falhas mapeadas. Para se estabelecer a correlação entre a atividade sísmica intraplaca e a geologia de uma dada área de estudo é necessário um vasto conjunto de dados sismológicos, geológicos e geofísicos. Espera-se que novos dados geológicos e geofísicos provenientes de novos projetos, tais como o do INCT de Estudos Tectônicos, possibilitem, quando integrados, uma melhor explicação para a atividade sísmica aqui estudada.

\section{Agradecimentos}

Os Autores agradecem aos técnicos do LabSls pela instalação, operação e manutenção da rede de estações localizada na região de Sobral-CE. Ao Dr. Marcelo Assumpção, do IAG/USP, pela ajuda na confecção dos mecanismos focais.

\section{Referências}

ALMEIDA, F.F.M, HASUI, Y., NEVES, B.B.B, FUCK, R.A. 1981. Brazilian structural provinces: an introduction. Earth Sci. Rev. 17:20-34.

ASSUMPÇÃO, M. 1992. The Regional intraplate stress field in South América. J. Geophys.Res., 97(B8): 1188911903.

BEZERRA, F.H.R., FERREIRA, J.M., SOUSA, M.O.L. 2006. Review of Seismicity and Neogene Tectonics in Northeastern Brazil. Rev. Assoc. Geol. Argentina. 61 (4): 525-535.

BORMANN, P., BAUMBACH, M., BOCK, G., GROSSER, H., CHOY, G.L AND BOATWRIGHT. 2002. IASPEI - New Manual of Seismological Observatory Practice. Vol 1.

CHARMICHAEL, R.S. 1982. Handbook of Physical Properties of Rocks, vol. II. CRS Press, 345 pp.

DNPM, 1973. Projeto Jaibaras. 1:100,000, DNPM, Brasil. FENG, M., ASSUMPÇÃO, M., VAN DER LEE, S. 2004. Group-Velocity Tomography and Lithospheric S-Velocity of South American Continent. Physics of the Earth and Planetary Interiors. 147: 315-331.

FERREIRA, J.M., OLIVEIRA, R.T., TAKEYA, M.K. \& ASSUMPÇÃO, M. 1998. Superposition of local and regional stress in northeast Brazil: evidence from focal mechanism around the Potiguar marginal basin. Geophys. J. Int., 134: 341-355.

FRANÇA, G.S., FERREIRA, J.M., TAKEYA, M.K. 2004. Seismic Activity in Senador Sá - CE, Brazil, 1997-1998. Rev. Brás. Geofís. 22(2): 115-125.

KISSSLINGER, C. \& ENGDAHL, E.R. 1973. The interpretation of the Wadati diagram with relaxed assumptions. Bull.Seism.Soc.Am. 63: 1723-1736.

LEE, W.H.K. \& LAHR, J.C. 1975. HYPO71 (revised): a computer program for determining hypocenter, magnitude and first motion pattern of local earthquakes. U.S. Geol. Surv. Open File Rep. 75-311, 114 pp.

REASENBERG, P. \& D. OPPENHEIMER. 1985. FPFIT, FPPLOT and FPPAGE: Fortran computer programs for calculating and displaying earthquake fault-plane solutions. U.S. Geol. Surv. Open File Rep. 85-739, 109 pp.

TAPLEY W.C. \& TULL. J.E. 1991. SAC-Seismic Analysis Code, USER MANUAL. Livermore National Laboratory, $413 \mathrm{pp}$.

SANTOS, T.J.S., FETTER, A.H, HACKSPACHER, P.C., NOGUEIRA NETO, J.A. 2008. Neoproterozoic Tectonic and Magmatic Episodes in the NW Sector of Borborema Province, NE Brazil, During Assembly of Western Gondwana. Journal of South American Earth Sciences. 25: 271-284. 